Andrade-Abarca, P.S.; Ramón-Jaramillo, L.N. y Loaiza-Aguirre, M.I. (2018). Aplicación del SEEQ como instrumento para evaluar la actividad docente universitaria. Revista de Investigación Educativa, 36(1), 259-275.

DOI: http://dx.doi.org/10.6018/rie.36.1.260741

\title{
Aplicación del SEEQ como instrumento para evaluar la actividad docente universitaria
}

\section{Application of the SEEQ as an instrument to evaluate university teaching activity}

\author{
Paola S.Andrade-Abarca, Lizeth N. Ramón-Jaramillo y María I. Loaiza-Aguirre \\ Ciencias Empresariales, Área Administrativa, Universidad Técnica Particular de Loja (Ecuador)
}

\section{Resumen}

En la presente investigación se aplicó el cuestionario "Student Evaluation of Educational Quality" (SEEQ) en el contexto latinoamericano, con el objetivo de conocer la percepción de los estudiantes acerca de la actividad docente universitaria. Se utilizó el método cuantitativo para realizar una medición minuciosa de las variables a través del análisis descriptivo e inferencial univariado. La muestra se conformó por 820 estudiantes de una universidad latinoamericana, quienes se encontraban cursando diferentes años en diversas áreas de saberes. Se confirmó la confiabilidad del instrumento al aplicarlo en un contexto diferente al que fue desarrollado. Los principales resultados muestran que: (1) los profesores universitarios se destacan en las dimensiones de aprendizaje y tarea, (2) los estudiantes perciben que el estímulo, motivación y suministro de lecturas y tareas mejoran su aprendizaje y (3) se presentaron deficiencias del profesorado en habilidades de tipo social, siendo poco visibles la actitud del docente para relacionarse con los alumnos y la habilidad para atraer su atención. Dadas las asociaciones que se presentaron entre las dimensiones evaluadas, las debilidades pueden ser mejoradas mediante el desarrollo de estrategias encaminadas a la formación de los profesores desde una perspectiva integral del proceso de enseñanza-aprendizaje.

Palabras clave: criterio de evaluación; evaluación del profesor; SEEQ; universidad.

Correspondencia: Paola S. Andrade-Abarca, Departamento de Ciencias Empresariales, Universidad Técnica Particular de Loja, Loja, PC. 110111, Ecuador. E-mail: psandrade@utpl.edu.ec. 


\begin{abstract}
This research has applied the questionnaire "Student Evaluation of Educational Quality" (SEEQ) in the Latin American context, in order to know the perception of students about the university teaching activity. The quantitative method was used to perform a careful measurement of the variables, using a descriptive and inferential analysis. The sample was composed of 820 students in a Latin American university, who were enrolled in different courses and areas of knowledge. The reliability of the instrument has been checked by applying it in a different context from the one where it was developed. The main results show that: (1) university professors have a better performance in the learning and assignment dimensions; (2) students perceive that encouragement, motivation and assignments improve their learning; and (3) weaknesses have been identified in teachers' social skills, since their abilities to interact with students and attract their attention have been rated low. On the basis of the connections detected among the assessed dimensions, these weaknesses can be overcome by developing strategies for the training of teachers from a holistic perspective of the teaching-learning process.

Keywords: evaluation criterion; teacher evaluation; SEEQ; university.
\end{abstract}

\title{
Introducción
}

Independientemente, del enfoque de enseñanza que caracteriza al profesorado o a una institución de educación superior, es importante evaluar la actividad docente. Los procesos de evaluación de la docencia resultan ineludibles y son prácticas utilizadas en la mayoría de universidades.

Son múltiples las finalidades de la evaluación docente, para Marsh y Roche (1997) tiene dos propósitos: a) desde el plano institucional pretende medir el grado de satisfacción del alumnado con las prácticas docentes, con el fin de ofrecer servicios educativos de calidad y establecer criterios precisos para la promoción del profesorado; y, b) en un nivel instruccional se busca incrementar la efectividad de la docencia para mejorar el proceso de enseñanza-aprendizaje.

Para Villa (2008) la evaluación de la actividad docente ha tenido dos propósitos fundamentales: mejorar la calidad de enseñanza y aportar en la rendición de cuentas y toma de decisiones en una universidad.

Además de los propósitos de la evaluación docente, esta debe contemplar una amplia gama de actividades como la docencia, asesoría, tutoría, difusión cultural, elaboración de materiales didácticos y la investigación (Cárdenas, Méndez \& González, 2014).

En relación a la calidad de la enseñanza, Adams et al. (2015) mencionan que el principal propósito de un sistema de evaluación debe ser el mejoramiento de la enseñanza para promover un mayor aprendizaje en el estudiante, recomiendan un sistema coherente y confiable de evaluación docente basado en estándares profesionales y sofisticados, suficientes para evaluar la calidad de la enseñanza como el desarrollo continuo del profesor, generando retroalimentaciones útiles en el proceso.

Aunque los sistemas de evaluación docente tienen diferentes orígenes, motivaciones y objetivos de acuerdo al contexto de políticas de cada país, por lo general buscan recolectar información sobre aspectos diversos de la práctica docente dentro y fuera del aula. Estos sistemas identifican a los maestros con problemas para 
intervenir y ayudarlos a mejorar su enseñanza y sus prácticas en clases a través de medidas correctivas, o contrariamente, para brindar incentivos a los mejores maestros (Martínez, 2013).

Existen dos tipos de evaluaciones, sumativa y formativa. Los aspectos sumativos, por sí solos, no inciden necesariamente en la actividad docente, por lo que es imprescindible la evaluación formativa para detectar fortalezas y debilidades orientadas a reflexiones y retroalimentaciones para mejorar lo que no funciona de forma adecuada (Verdugo \& Cal, 2010).

La evaluación de la actividad docente bajo el enfoque formativo ayuda a los docentes a diagnosticar falencias propias y a mejorarlas a partir de acciones de formación que incidan en su desarrollo profesional. Martínez (2013) menciona que la evaluación docente implica identificar a los maestros con problemas para intervenir y ayudarlos a mejorar su enseñanza y sus prácticas en clases a través de medidas correctivas, o contrariamente, para brindar incentivos a los mejores maestros.

Se utiliza una variedad de enfoques para recolectar información de cada constructo o aspecto que describa la calidad o el desempeño docente (Martínez, 2013). Salazar (2008) identifica cuatro actores que participan en el proceso de evaluación docente: a) estudiantes, por medio de una encuesta de opinión; b) docente, mediante una encuesta en la que se registra su autoevaluación; c) jefe directo, a través de su evaluación; y, d) pares, mediante una encuesta de evaluación.

Desde la perspectiva de quien realiza la evaluación Rodríguez e Ibarra (2011) manifiestan que se puede diferenciar la autoevaluación, la cual es una evaluación realizada por el propio docente y la heteroevaluación llevada a cabo por otros.

De los enfoques mencionados, por lo general predominan las encuestas de opinión estudiantil, siendo las más utilizadas en las instituciones de educación superior (Rueda \& Luna, 2011), teniendo su desarrollo en el último tercio del siglo XX (Villa, 2008). Se considera a los estudiantes como la mejor fuente de información del proceso de enseñanza aprendizaje, ya que son los actores directos y pueden referenciar sobre el desarrollo de la actividad de sus docentes en el aula (Tirado, Miranda \& Sánchez, 2007).

Hay que reconocer que la evaluación de la docencia universitaria a través de los cuestionarios de los estudiantes, y la evaluación institucional han supuesto la antesala para introducir los sistemas de garantía interna de calidad universitaria (Villa, 2008, p. 187).

Considerando los diferentes actores que intervienen en la evaluación docente, la orientación del presente trabajo se enfoca en la percepción de los estudiantes sobre la actividad docente como un criterio válido para la mejora académica.

De acuerdo a Verdugo y Cal (2010) las encuestas de opinión son una práctica habitual de evaluación docente asumida en la enseñanza superior. Además el uso de la encuesta puede aportar datos que sirvan para diseñar mejoras significativas en la práctica docente (Corral, Almajano \& Domingo, 2008).

Adicionalmente, las encuestas a estudiantes tienen una menor influencia del contexto social y pueden ser usadas para crear indicadores confiables al nivel del aula 
(Kane \& Staiger, 2012; Martínez, 2013). Cortés, Campos y Moreno (2014) recomiendan el uso de estos cuestionarios para mejorar la enseñanza, la toma de decisiones sobre el personal académico y salvaguardar el control de la calidad educativa.

A pesar de sus ventajas, se evidencian críticas aplicadas a la mayoría de los sistemas de evaluación docente, percibidos como rituales monótonos sin consecuencias ni utilidad y con poco valor ya sea sumativo, formativo o informativo (Glazerman et al., 2010). En este sentido Reyero (2014) considera que en ocasiones la estadística resulta excesiva y que no todo puede medirse con instrumentos cuantitativos.

Además, hay que considerar que la mayoría de los instrumentos que valoran la actividad docente a través de la opinión estudiantil miden la actividad del profesor centrada en la enseñanza más que en el aprendizaje de los estudiantes (Villa, 2008).

La percepción de los estudiantes como criterio de evaluación también ha sido cuestionada, estudios de Galbraith y Merrill (2012) indican que los colegas identifican de forma mucho más precisa a los mejores docentes que los estudiantes.

A pesar de las críticas encontrados en varios estudios, por medio de los resultados se observa que el cuestionario es el instrumento más común y adecuado para evaluar la actividad docente por parte de los estudiantes.

\section{Cuestionario “Student Evaluation of Educational Quality” (SEEQ)}

El cuestionario Student Evaluation of Educational Quality (SEEQ) es un instrumento que permite analizar la eficiencia de la enseñanza utilizando nueve dimensiones: aprendizaje, entusiasmo, organización, interacción con los estudiantes, actitud personal, contenidos, evaluación, tareas y una valoración general; las cuales están compuestas por varios ítems, valorados en una escala tipo Likert. Su uso está fundamentado en sus propiedades psicométricas, aplicabilidad en varios contextos del ámbito académico y permite la posibilidad de perfeccionar los ítems de cada dimensión (Verdugo \& Cal, 2010). Este instrumento fue diseñado por Herbert Marsh en 1982 y constituye uno de los cuestionarios mayormente utilizados en la evaluación de la enseñanza por parte de los estudiantes (Al-Muslim y Arifin, 2015).

La percepción de los estudiantes sobre la actividad docente, y la existencia de un sistema de evaluación permiten la mejora continua de la calidad educativa y la detección de áreas de oportunidad en su práctica (Olavarrieta, Gomez \& García, 2015). Los autores concluyen que los profesores perciben al SEEQ como un instrumento útil para mejorar el sistema de evaluación docente, por lo que es positivo que una universidad considere su aplicación.

España fue el primer país iberoamericano en hacer investigación con el SEEQ (García \& Medécigo, 2014). Adaptaciones en el mismo contexto fueron realizadas por Corral et al. (2008) y Pérez-Poch (2006) en la Universidad Politécnica de Catalunya; en la Universidad de Vigo por Bol, Pérez y Sáiz (2013) y Verdugo y Cal (2010) en la Universidad de Burgos.

A partir de la perspectiva de la presente investigación, se pretende considerar los resultados del SEEQ con una visión evaluativa formativa, que permita valorar la función del docente universitario en un contexto latinoamericano, para mejorar el proceso de enseñanza-aprendizaje a través de estrategias de refuerzo a su actividad. 


\section{Objetivos de la investigación}

En esta investigación se propone en primer lugar conocer la percepción de los estudiantes sobre la actividad del docente universitario, a través de la aplicación del instrumento Student Evaluation of Educational Quality (SEEQ), en segundo lugar, se analizará las relaciones existentes entre las dimensiones propuestas por el SEEQ con el perfil del estudiante y las características académicas de la materia evaluada (tipo, dificultad, carga de trabajo y horas de dedicación semanal). Finalmente se analiza la existencia de asociaciones entre las dimensiones propuestas en el SEEQ.

\section{Método}

La presente investigación partió de una adaptación del SEEQ al contexto latinoamericano ya que esta herramienta ha sido principalmente aplicada a estudiantes cuya lengua materna ha sido la inglesa.

Para esta adaptación se inició con su transcripción del inglés al castellano a través de una traducción lineal (Carvajal, Centeno, Watson, Martínez \& Sanz, 2011). Con la aplicación de la versión en español del instrumento a través de un sistema en línea y la obtención de los resultados se comprobó la fiabilidad del instrumento mediante un análisis de confiabilidad y factorial confirmatorio (AFC) (Ochoa-Meza, Sierra, PérezRodrigo \& Aranceta-Bartrina, 2014).

A partir de los resultados de la fiabilidad del instrumento se procedió a la aplicación en la muestra seleccionada. Para el análisis de los resultados se llevó a cabo un análisis descriptivo, mediante el cual se pretende determinar las características de un conjunto de observaciones; $\mathrm{y}$, un análisis inferencial univariado para contrastar las relaciones entre dos variables o grupo de observaciones (Instituto de Perfeccionamiento y Estudios Superiores, 2009). Este método cuantitativo permitió realizar una medición minuciosa de las variables sobre la base de objetivos bien definidos y delimitados (Corona-Lisboa, 2016).

\section{Población y Muestra}

Se consideró como población objetivo la comunidad estudiantil de grado de una universidad latinoamericana constituida por 5111 estudiantes matriculados en el 2014, quienes en su totalidad fueron invitados a contestar el instrumento, de esta población 2787 estudiantes contestaron la encuesta. Se aplicó un proceso de depuración obteniéndose 820 respuestas válidas, las que constituyeron la muestra final productora de datos, con un error muestral del $3.20 \%$.

Los estudiantes se encontraban cursando años diferentes en sus carreras, y evaluaron a un grupo de materias tipificadas como genéricas (asignaturas consideradas como pilares de la profesión y contribuyen a adquirir competencias genéricas) y troncales (asignaturas que proporcionan contenidos específicos y propios de la carrera a través de los cuales se alcanzan competencias específicas) dentro de la estructura académica de la universidad objeto de estudio. El 50.4\% de la muestra son hombres y el $49.6 \%$ mujeres, este grupo de alumnos forman parte de 23 carreras de grado de diferentes 
áreas de saberes; el $6.4 \%$ se encontraba cursando su primer año de carrera, $24.9 \%$ el segundo año, $18.7 \%$ el tercer año, $19.9 \%$ cuarto año, y el 30.1\% el quinto año. Finalmente, el $21.9 \%$ de los alumnos evaluaron una asignatura de tipo genérica y el $78.1 \%$ una troncal.

\section{Instrumento}

El SEEQ es un instrumento que valora la efectividad de la enseñanza y se encuentra conformado por 31 ítems valorados en una escala likert de 1 Totalmente en desacuerdo a 5 Totalmente de acuerdo, agrupados en 9 dimensiones:

- Aprendizaje: mide si los estudiantes encontraron las clases estimulantes y sus intereses aumentaron en la asignatura.

- Entusiasmo: evalúa la actitud del profesor y su habilidad para atraer la atención de los estudiantes con la pedagogía usada en clase.

- Organización: mide si el docente alinea los objetivos con los materiales de la asignatura y si están preparados y presentados apropiadamente.

- Interacción con los estudiantes: evalúa la habilidad del profesor para motivar a los estudiantes a participar de discusiones en clase.

- Actitud personal: mide la habilidad del profesor para relacionarse con los alumnos a través de buenas técnicas de relación social y ser accesible durante horas de oficina y después de clase.

- Contenido: evalúa si el profesor realiza comparaciones y contrastes de ideas, presenta puntos de vista diferentes a los suyos, tendencias y descubrimientos.

- Evaluación: mide si el método de evaluación es justo, apropiado y alineado con los contenidos y si el docente realiza retroalimentaciones.

- Tareas: evalúa la habilidad del profesor para proveer lecturas y tareas que contribuyan a entender la asignatura.

- Valoración general: evalúa al profesor y la asignatura de manera integral.

\section{Procedimiento de recogida y análisis de datos}

Posterior a la adaptación del SEEQ se procedió con la aplicación en línea del instrumento en un mismo momento temporal del ciclo académico. Con el fin de obtener mayor participación de estudiantes, la encuesta fue enviada a la totalidad de la población objetivo a través de sus direcciones de correo electrónico institucional, mediante la plataforma digital e-encuesta, durante el mes de junio del año 2014.

Para motivar las respuestas de los estudiantes, se enviaron recordatorios personalizados tres días de cada semana durante el mes que estuvo disponible el cuestionario en la plataforma. La participación de los estudiantes fue voluntaria, obteniéndose 2787 registros. Previo al análisis de estos datos, se depuró la información a través de la detección de duplicados y encuestas que registraron un grupo pequeño de preguntas (menos del 70\% de respuestas), posterior a ello se eliminaron dichas encuestas con el objetivo de no afectar los resultados generados, contando con 820 respuestas válidas, que constituyeron la muestra a estudiar. 
Finalmente, para el procesamiento de los datos recolectados se utilizó el paquete estadístico Statistical Package for the Social Sciences (SPSS) en su versión 22.0.

\section{Resultados}

\section{Adaptación y fiabilidad del SEEQ}

Respecto a la fiabilidad del instrumento, los resultados muestran un índice de confiabilidad de .972, el cual indica muy alta consistencia Cohen, Manion y Morrison (2013). En este mismo valor de lectura se encuentran las dimensiones de entusiasmo (.949), interacción con los estudiantes (.926) y contenidos (.908). Se presenta alta consistencia en las dimensiones de organización (.890), aprendizaje (.885), evaluación (.865) y tarea (.831). Finalmente, las dimensiones de actitud personal (.792) y valoración general (.770) registran una consistencia moderada.

El análisis factorial confirmatorio (AFC) muestra que la prueba de adecuación muestral $(\mathrm{KMO}=.979)$ y la prueba de esfericidad de Bartlett $\left(X^{2}=26947.53 ; \mathrm{p}<.001\right)$ presentan adecuación de los datos para el análisis. El AFC se realizó considerando las 9 dimensiones, las cuales explican el 79.6\% de la varianza total, el porcentaje de la varianza de los 3 primeros factores se encuentra en un rango del 11\% al 20\%, mientras que el porcentaje de la varianza explicada desde el cuarto factor es inferior al $10 \%$ (rango de 3.2\% a 8.6\%). Finalmente, las cargas factoriales de los ítems se distribuyen entre .378 y .937 .

\section{Percepción de los estudiantes sobre la actividad del docente universitario}

Los resultados de la evaluación de los estudiantes a las dimensiones del SEEQ (ver Tabla 1) muestran que aproximadamente el 70\% están "de acuerdo o totalmente de acuerdo" con el cumplimiento de las nueve dimensiones, destacándose los factores de aprendizaje $(73 \%)$ y tarea $(74 \%)$.

Las dimensiones con mayor porcentaje en las escalas "en desacuerdo, totalmente en desacuerdo y neutro" son: entusiasmo, organización, interacción con los estudiantes, actitud personal, contenido y evaluación, en estas dimensiones se evaluarán los porcentajes considerando la composición por ítems.

En la dimensión entusiasmo, el 61\% de los estudiantes indican estar "de acuerdo o totalmente de acuerdo" con la actitud del profesor y la capacidad para atraer la atención con su método de enseñanza; sin embargo, el 22\% manifiesta que el profesor no es entusiasta ni posee adecuada motivación, el 17\% de los alumnos tiene una opinión neutral sobre esta dimensión.

En el factor organización, el 65\% de los estudiantes sostienen que los materiales de la asignatura están preparados apropiadamente y se relacionan con los objetivos de la materia. Contrariamente a esta percepción, el 16\% manifiesta que las explicaciones no son claras, los materiales de las asignaturas son poco preparados y el plan docente no está relacionado con lo que se enseña en la clase. El 19\% restante muestra no estar de acuerdo o en desacuerdo con este aspecto. 
El 67\% de los alumnos están de acuerdo con la habilidad de los profesores para incentivar a los estudiantes a participar en las discusiones en clase, expresar sus propias ideas, cuestionar al profesor, hacer preguntas y recibir respuestas completas y significativas; mientras que el 15\% no está de acuerdo con esta opinión. Finalmente, el 18\% tiene una apreciación neutra acerca de la dimensión de interacción con los estudiantes.

En cuanto a la actitud personal de los docentes, el $62 \%$ de los alumnos consideran que los profesores poseen habilidades para relacionarse con los alumnos a través de técnicas de relación social y son accesible durante horas de oficina y después de clase.; contrariamente el 18\% manifiesta lo contrario. El 20\% de los alumnos se ubican en un nivel neutro.

En la dimensión contenido el $64 \%$ de los estudiantes responden que los profesores discuten sus puntos de vista y los comparan con teorías a través de su método de enseñanza; el 15\% señala que los profesores no realizan comparaciones ni contrasta ideas o teorías y se evidencia carencia de tendencias y descubrimientos en el campo de estudio de la asignatura. Finalmente, la opinión del 21\% es neutra.

En la dimensión evaluación el $67 \%$ de los alumnos manifiestan que la retroalimentación y comentarios del profesor con respecto a los exámenes y deberes calificados es valiosa; los métodos de evaluación son justos y apropiados para la asignatura; y, los exámenes y trabajos calificados se basan en los contenidos que desarrolló el profesor. Por otra parte, se evidencia que el $15 \%$ de los alumnos consideran que no se detecta calidad, igualdad, valor y oportuna retroalimentación de los profesores en función de los objetivos planteados en las materias y su relación con las evaluaciones. El 18\% restante mantiene una percepción neutra.

Tabla 1

Porcentajes por dimensiones del SEEQ

\begin{tabular}{lccccc}
\hline \multicolumn{1}{c}{ Dimensiones } & $\begin{array}{c}\text { Totalmente } \\
\text { en } \\
\text { desacuerdo }\end{array}$ & $\begin{array}{c}\text { En } \\
\text { desacuerdo }\end{array}$ & Neutro & $\begin{array}{c}\text { De } \\
\text { acuerdo }\end{array}$ & $\begin{array}{c}\text { Totalmente } \\
\text { de acuerdo }\end{array}$ \\
\hline Aprendizaje & $5 \%$ & $6 \%$ & $16 \%$ & $30 \%$ & $43 \%$ \\
Entusiasmo & $11 \%$ & $10 \%$ & $17 \%$ & $27 \%$ & $34 \%$ \\
Organización & $8 \%$ & $8 \%$ & $19 \%$ & $28 \%$ & $37 \%$ \\
Interacción con los & $7 \%$ & $8 \%$ & $18 \%$ & $29 \%$ & $38 \%$ \\
estudiantes & $9 \%$ & $9 \%$ & $20 \%$ & $28 \%$ & $34 \%$ \\
Actitud personal & $7 \%$ & $8 \%$ & $21 \%$ & $30 \%$ & $34 \%$ \\
Contenido & $7 \%$ & $8 \%$ & $18 \%$ & $31 \%$ & $36 \%$ \\
Evaluación & $5 \%$ & $5 \%$ & $16 \%$ & $30 \%$ & $44 \%$ \\
Tarea & $8 \%$ & $8 \%$ & $18 \%$ & $28 \%$ & $38 \%$ \\
Valoración general & & & & & \\
\hline
\end{tabular}




\section{Asociaciones entre dimensiones y características académicas}

Existen diferentes asociaciones entre las dimensiones que componen el SEEQ con las variables que describen el perfil del estudiante y características académicas (ver Tabla 2).

Tabla 2

Asociación entre las dimensiones del SEEQ y características de los estudiantes

\begin{tabular}{|c|c|c|c|c|c|c|c|}
\hline Dimension & & Sexo & Semestre* & $\begin{array}{l}\text { Tipo de } \\
\text { materia }\end{array}$ & $\begin{array}{l}\text { Dificultad } \\
\text { de materia }\end{array}$ & $\begin{array}{l}\text { Carga de } \\
\text { trabajo de }\end{array}$ & $\begin{array}{l}\text { Horas de } \\
\text { dedicación }\end{array}$ \\
\hline & $\mathrm{X}^{2}$ & & & 61.45 & 125.04 & 157.58 & 181.20 \\
\hline Aprendizaje & Sig. & & & .027 & .002 & .001 & .001 \\
\hline Fntusiasmo & $\mathrm{X}^{2}$ & & & & & 180.31 & \\
\hline - & Sig. & & & & & .000 & \\
\hline Oroanización & $X^{2}$ & & 201.99 & & 162.42 & 222.84 & 209.89 \\
\hline Uigantizaciont & Sig. & & .000 & & .000 & .000 & .000 \\
\hline Interacción & $X^{2}$ & & 153.13 & 152.54 & 119.46 & 157.07 & 170.41 \\
\hline $\begin{array}{c}\text { con los } \\
\text { estudiantes }\end{array}$ & Sig. & & .002 & .000 & .007 & .001 & .005 \\
\hline Actitud & $\mathrm{X}^{2}$ & & 143.91 & 58.57 & & & 163.06 \\
\hline personal & Sig. & & .007 & .046 & & & .015 \\
\hline Contenidos & $\mathrm{X}^{2}$ & & & & 167.10 & & 189.14 \\
\hline comientaos & Sig. & & & & .000 & & .007 \\
\hline Fylurionec & $\mathrm{X}^{2}$ & 34.51 & 243.45 & & 103.52 & 173.91 & 169.83 \\
\hline Lvaluaciones & Sig. & .007 & .000 & & .004 & .000 & .000 \\
\hline Tareas & $\mathrm{X}^{2}$ & 21.75 & 97.19 & & 65.01 & 107.11 & 122.97 \\
\hline Iareas & Sig. & .010 & .000 & & .002 & .000 & .000 \\
\hline
\end{tabular}

* Esta información hace referencia al semestre de estudio en el que se ubica el alumno

Las asociaciones significativas entre el sexo de los alumnos con las dimensiones evaluación $\left(\mathrm{X}^{2}=34.51 ; \mathrm{p}<.005\right)$ y tarea $\left(\mathrm{X}^{2}=21.75 ; \mathrm{p}<.005\right)$ indican que las mujeres tienden a valorar mejor al profesor que los hombres.

La relación detectada entre el semestre de estudio de los alumnos y las dimensiones del SEEQ: organización $\left(X^{2}=201.99 ; p<.005\right)$, interacción con los estudiantes $\left(X^{2}=153.13\right.$; $\mathrm{p}<.005)$, actitud personal $\left(X^{2}=143.91 ; \mathrm{p}<.005\right)$, evaluación $\left(X^{2}=243.45 ; \mathrm{p}<.005\right)$ y tarea $\left(X^{2}=97.19 ; p<.005\right)$ reflejan que los estudiantes que se encuentran iniciando su carrera universitaria (primer semestre) son más indulgentes al momento de evaluar estos aspectos. Únicamente, en la dimensión actitud personal se evidencia que, a partir del sexto semestre, los estudiantes tienden a valorar mejor a la habilidad del profesor para proveer espacios considerando las diferencias individuales y el acompañamiento proporcionado. 
Las dimensiones aprendizaje $\left(X^{2}=61.45 ; \mathrm{p}<.005\right)$, interacción con los estudiantes $\left(X^{2}=152.54 ; \mathrm{p}<.005\right)$ y actitud personal $\left(X^{2}=58.57 ; \mathrm{p}<.005\right)$ presentan asociación con el tipo de materia, estas relaciones muestran que los alumnos tienen una mejor percepción de las actitudes y habilidades de los profesores cuando estos imparten materias troncales o de contenido disciplinar.

Finalmente, las dimensiones aprendizaje, organización, interacción con los estudiantes, evaluación y tarea están asociadas con la dificultad de la materia, su carga de trabajo y las horas de dedicación del estudiante a la semana. Mientras que, entusiasmo se relaciona estadísticamente con la carga de trabajo de la materia; actitud personal está asociada con las horas de dedicación del estudiante; y, la dimensión contenidos se relaciona con la dificultad de la materia y las horas de dedicación del alumno.

\section{Asociaciones entre dimensiones}

Las relaciones existentes entre las dimensiones del SEEQ muestran correlaciones significativas, positivas moderadas y fuertes (Aguayo y Lora, 2007). Se evidencia 9 asociaciones moderadas y 19 relaciones positivas fuertes, las cuales se muestran en la Figura 1.
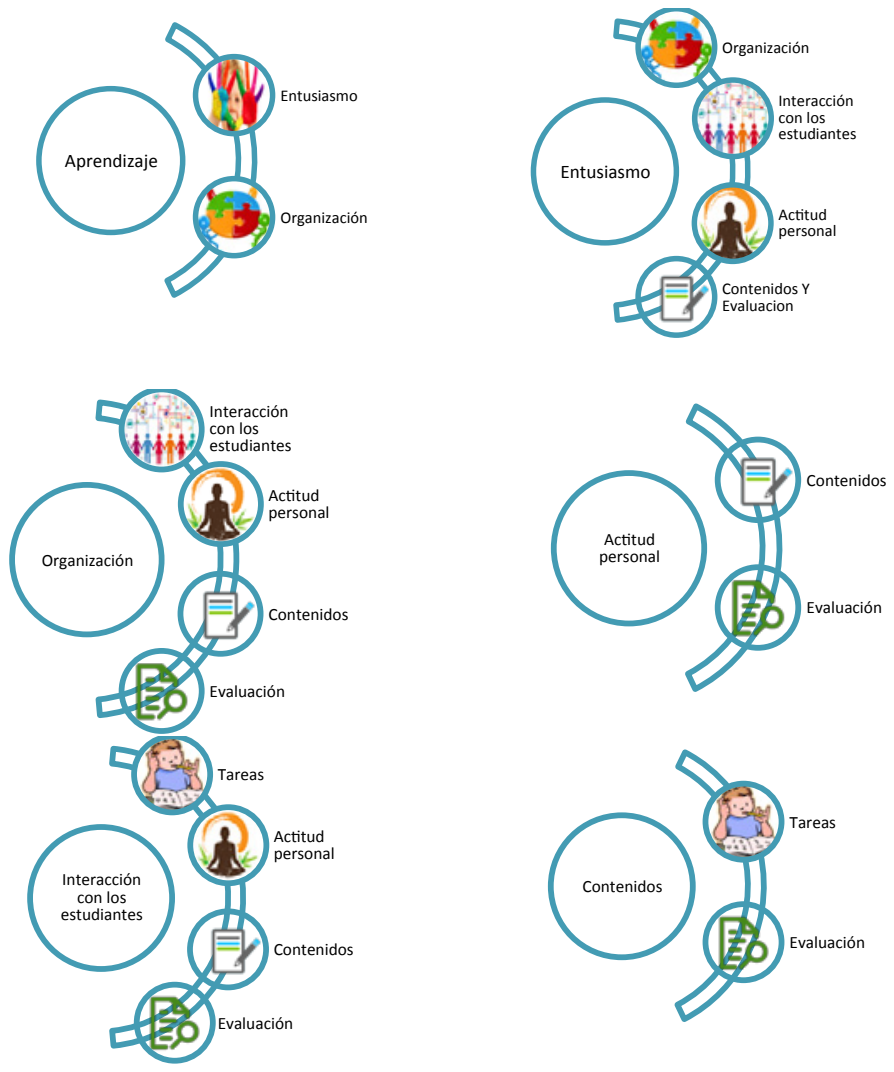

Figura 1. Correlaciones fuertes entre dimensiones. 
La organización del profesor está fuertemente relacionada con: la interacción con los estudiantes ( $\mathrm{rho}=.827 ; \mathrm{p}<.001)$, actitud personal ( $\mathrm{rho}=0.746 ; \mathrm{p}<.001$ ), contenidos (rho=0.815; $\mathrm{p}<.001) ; \mathrm{y}$, evaluación (rho=0.804; $\mathrm{p}<.001$ ).

La dimensión interacción con los estudiantes se encuentra fuertemente relacionada con: la actitud personal del profesor ( $r h o=0.746 ; \mathrm{p}<0.001)$, contenidos (rho=.796; $\mathrm{p}<.001$ ), evaluación (rho=.761; $\mathrm{p}<.001)$ y tareas (rho=.712; $\mathrm{p}<.001$.

El entusiasmo que trasmite el profesor y su habilidad para atraer la atención de los estudiantes se encuentra asociada con: la organización (rho=.866; $\mathrm{p}<.001$ ), habilidad del profesor para incentivar a los estudiantes a expresar sus propias ideas (rho=.813; $\mathrm{p}<.001)$, proveer espacios tomando en consideración las diferencias individuales ( $\mathrm{rho}=.778$; $\mathrm{p}<.001)$, habilidad del profesor para discutir los puntos de vista de los estudiantes (rho=.794; $\mathrm{p}<.001) ; \mathrm{y}$, la evaluación ( $\mathrm{rho}=.772 ; \mathrm{p}<.001)$.

Otra fuerte relación es la que se encuentra entre la actitud personal del profesor ( $\mathrm{rho}=.750 ; \mathrm{p}<.001)$, contenidos y evaluaciones (rho=.720; $\mathrm{p}<.001)$. Así como, contenidos con evaluaciones $(\mathrm{rho}=.825 ; \mathrm{p}<.001)$, y las tareas planificadas por el profesor $(\mathrm{rho}=.711$; $\mathrm{p}<.001)$.

\section{Discusión y conclusiones}

Los resultados encontrados coinciden con lo presentado en otras investigaciones, ya que el instrumento muestra fiabilidad al momento de aplicarlo en un contexto latinoamericano y permite evaluar la actividad del docente relacionándola con las características de la materia (Marsh, 1987; Coffey \& Gibbs, 2001; Olavarrieta et al., 2015).

Se exploró la fiabilidad del SEEQ mediante la consistencia interna, cuya estimación se efectuó al aplicar la prueba de Alfa de Cronbach al instrumento en su totalidad y a cada una de las dimensiones. En este sentido, Cohen et al. (2013) establecen un alfa de .7 como consistencia moderada. En el presente estudio, las 9 dimensiones mostraron índices de confiabilidad superiores a .7 y un Alfa de Cronbach de .972 en el cuestionario, coincidiendo con los resultados obtenidos por Bol et al. (2013) quienes lograron un índice de confiabilidad de .92.

Con la finalidad de analizar la validez de la escala se efectuó un análisis factorial de componentes principales por rotación varimax normalizada, a partir de las puntuaciones de los 31 ítems del instrumento para comprobar a qué dimensiones pertenecen los ítems; se detectan 3 dimensiones que explican el 20\% de la varianza, mientras que Bol et al. (2013) encontraron que dos factores explican el $65.60 \%$ de la varianza. Además, se observa que las nueve dimensiones de la escala del SEEQ logran explicar el 79.6\% de la varianza.

Los resultados de esta investigación muestran que las dimensiones de aprendizaje y tarea son mejor evaluadas por los estudiantes, corroborando los resultados de Clemente y Gómez (2014) quienes muestran que en aprendizaje y tareas el 78.6\% y 65\% de los estudiantes valoran con 5 y 4 puntos, respectivamente. También Solé-Pareta, Domingo-Pascual y Santos-Boada (2014) manifiestan que el factor aprendizaje es uno de los mejor valorados, exceptuando tareas.

El entusiasmo y la actitud personal del profesor son dimensiones que han sido valoradas por los estudiantes con los porcentajes más altos en las escalas de "total- 
mente en desacuerdo, en desacuerdo y neutro". De igual manera estas apreciaciones se evidencian en investigaciones como la de Marsh y Roche (1997) quienes indican que existen algunos perfiles docentes que pueden tener una alta puntuación en organización pero baja en entusiasmo. Esto se puede justificar debido a que existe la idea por parte de los profesores que no se debe consultar a los estudiantes acerca del entusiasmo y humor del profesor, debido a que el docente se presenta a una clase no para "agradar a los alumnos, sino para tratar de formarlos" (Olavarrieta et al., 2015, p. 11).

En cuanto actitud personal, se registran resultados poco favorables, lo que contrariamente se demuestra en otros estudios en los que esta dimensión es bien percibida por los estudiantes. Clemente y Gómez (2014) señalan que aproximadamente el 80\% de los estudiantes están totalmente de acuerdo en la dimensión actitud personal; mientras que Solé-Pareta et al. (2014) mencionan que esta dimensión es una de las mejor valoradas.

Se detectó una relación fuerte significativa entre las dimensiones aprendizaje con entusiasmo y organización. Esto reafirma lo expuesto por Pintrich y Schunk (2002) y Pintrich (2003) quienes relacionan la motivación hacia las asignaturas y los resultados de aprendizaje; para estos autores es un hecho que si el profesor consigue despertar el interés de los alumnos por la materia, los estudiantes perciben el proceso de enseñanza-aprendizaje de manera positiva y presumiblemente habrá una mejora en los resultados académicos.

Situación similar se observa al relacionar la dimensión entusiasmo con organización, interacción, actitud del docente, contenidos y evaluación. Para Rama (2011) existen ciertas variables que permiten a los docentes ser más eficaces, entre ellas el conocimiento de las necesidades de sus estudiantes y por ende un aumento de su motivación, uso de diferentes estrategias de enseñanza, buenas habilidades comunicativas y trasmisión de optimismo al estudiante sobre sus capacidades personales. Soberón et al. (2015) mencionan en este sentido, que las habilidades de planificación docente y el dominio de la materia son importantes como lo son las habilidades sociales, siendo imprescindible una retroalimentación entre ellas.

Asimismo, se evidencian asociaciones fuertes entre la dimensión interacción con los estudiantes y actitud personal, contenidos, evaluaciones y tareas; y también en el factor actitud personal con las dimensiones de contenidos y evaluación. Se demuestra en investigaciones que el docente universitario debe ser capaz de interactuar con los estudiantes, de tal forma que apoye su crecimiento académico y personal. "Cuando los alumnos perciben que existe cierta afinidad y familiaridad, están más atentos y comprometidos con los procesos de clase" (Acevedo \& Fernández, 2004, p. 151).

La dimensión contenidos se asocia con evaluación y tareas, corroborando que la evaluación debe evolucionar hasta el punto de que forme parte del contenido curricular del aprendizaje (Bordas \& Cabrera, 2001). La importancia de estas dimensiones se ven traducidas en las competencias básicas que adquiere un docente, en la que los objetivos, contenidos, metodología y criterios de evaluación son el principal nivel de integración y concreción de dichas competencias (Bolívar, 2010). Por su parte, García-Señoran, González y Soto (2015, p. 258) asocian los términos tarea y contenido y mencionan "el interés personal por una tarea o contenido académico implica experimentar sentimientos positivos y atribuir un valor personal a esa tarea o contenido". 
Se detectó asociaciones significativas entre las dimensiones de evaluación y tareas con el sexo de los estudiantes, mostrando que las mujeres en mayor porcentaje valoran mejor al profesor. Estos resultados se asemejan al estudio llevado a cabo en la Universidad de Barcelona orientado a conocer la opinión de los alumnos sobre las competencias que debería tener un buen profesor, en el que se demostró que las mujeres otorgan puntuaciones más altas a competencias como metodología, planificación y buena transmisión de los contenidos (Santigosa, Costas, Campos \& Jorba, 2014).

Finalmente, se encontró asociación entre el semestre de estudio de los alumnos con organización, interacción con los estudiantes, actitud personal, evaluación y tareas. Pounder (2007) comprueba que las encuestas dirigidas a estudiantes funcionan mejor para medir la calidad del profesor en cursos más altos, situación que se evidencia en el factor actitud personal, en el que a partir del sexto semestre de estudio los alumnos tienden a valorarla mejor.

En función de los resultados logrados en la presente investigación, se propone cimentar estrategias de formación en cuatro dimensiones: entusiasmo, actitud personal, contenido y organización, las que de acuerdo a las respuestas dadas por los estudiantes han sido poco visibles en el quehacer docente.

Los resultados de esta formación se vislumbrarán en alinear los materiales de la asignatura a objetivos claros, manejar contenidos bien estructurados con opiniones fundamentadas, desarrollo de habilidades para atraer la atención de los estudiantes y mejorar la interacción con los alumnos en el ámbito social. Se esperaría que el perfeccionamiento de estas dimensiones incida en la mejora integral de la actividad docente, considerando la interrelación de las dimensiones abordadas en el SEEQ y por ende de los resultados logrados en su evaluación.

Los resultados dan muestra de la necesidad de implementar medidas de intervención en las universidades en los aspectos relacionados a la mejora en las competencias sociales y organizativas del docente, dichas medidas están en función de la creación o fortalecimiento de programas de formación y actualización docente, que tengan consecuencia directa en la planificación, preparación y desarrollo de la asignatura. Así lo corroboran García et al. (2004) quienes analizan que la evaluación debe tener un enfoque orientado al perfeccionamiento de los profesores procurando que la misma sirva para el desarrollo integral de la persona. Por ello, quienes se encargan de la evaluación docente cumplen un papel importante al reconocer y reenfocar los objetivos de los programas de evaluación para mejorar las prácticas de enseñanza-aprendizaje (Rueda, 2008).

Finalmente, es necesario además de la aplicación de instrumentos para evaluar la actividad docente universitaria con la participación del estudiante, implementar otras acciones que permitan valorar el proceso de enseñanza del profesorado resaltando puntos fuertes y débiles, de manera que los resultados sirvan a los tres protagonistas universitarios: (1) docentes, como insumos que orienten a realizar una autorreflexión de su actuación, (2) directivos para analizar la calidad de la enseñanza, organizar planes de formación e innovación y diseñar políticas y prácticas sobre la formación de profesores identificando modelos de enseñanza que se puedan replicar (3) a los estudiantes quienes serán partícipes de modelos de evaluación con fines de mejorar el proceso de formación. 


\section{Referencias}

Acevedo, R., \& Fernández, M. J. (2004). La percepción de los estudiantes universitarios en la medida de la competencia docente: Validación de una escala. Revista Educación, 28(2), 145-166.

Adams, T., Aguilar, E., Berg, E., Cismowski, L., Cody, A., Cohen, D., ... White, S. (2015). A coherent system of teacher evaluation for quality teaching. Education Policy Analysis Archives, 23(17), 1-26. doi: 10.14507/epaa.v23.2006

Aguayo, M., \& Lora, E. (2007). Cómo realizar "paso a paso" un contraste de hipótesis con SPSS para Windows: (III) Relación o asociación y análisis de la dependencia (o no) entre dos variables cuantitativas. Correlación y regresión lineal simple. Fundación Andaluza Beturia para la Investigación en Salud. Recuperado de http://www.fabis.org/ html/archivos/docuweb/contraste_hipotesis_3r.pdf

Al-Muslim, M., \& Arifin, Z. (2015). The Usability of SEEQ in Quality Evaluation of Arabic Secondary Education in Malaysia. Canadian Center of Science and Education, 8(3), 202-211.doi: 10.5539/ies.v8n3p202

Bol, A., Pérez, M., \& Sáiz, M. (2013). Validación de una encuesta de satisfacción de los estudiantes con la actividad docente: una propuesta de mejora en la Universidad de Burgos. $X$ Foro Internacional sobre la Evaluación de la Calidad de la Investigación y de la Educación Superior (FECIES). Granada. Recuperado dehttp://www.ugr.es/ aepc/ XIFECIESWEB/LIBRORESUMENESXFORO.pdf

Bolívar, A. (2010). Competencias básicas y currículo. Madrid: Síntesis.

Bordas, M., \& Cabrera, F. (2001). Estrategias de evaluación de los aprendizajes centrados en el proceso. Revista española de pedagogía, 218, 25-48.

Cárdenas, M., Méndez, L., \& González, M. (2014). Evaluation of teacher performance, stress and burnout in university professors. Actualidades Investigativas en Educación, 14(1), 93-114.

Carvajal, A., Centeno, C., Watson, R., Martínez, M., \& Sanz, A. (2011). ¿Cómo validar un instrumento de medida de la salud?. Anales del sistema sanitario de Navarra, 34(1), 63-72).

Casal, J., \& Mateu, E. (2003). Tipos de muestreo. Revista de Epidemiología y Medicina Preventiva, 1(1), 3-7.

Clemente, P., \& Gómez, A. (2014). Aplicación de un proceso de mejora continua en una asignatura de Desarrollo de Software Dirigido por Modelos. Trabajo presentado a en las XX Jornadas XX Jornadas de Enseñanza Universitaria de la Informática, Oviedo. Recuperado de http://upcommons.upc.edu/bitstream/handle/2099/15497/P355cl_apli. pdf? sequence $=1$ \&isAllowed $=\mathrm{y}$

Coffey, M., \& Gibbs G. (2001). The evaluation of the Student Evaluation of Educational Quality Questionnaire (SEEQ) in UK Higher Education. Assessment E Evaluation in Higher Education, 26(1), 89-93.

Cohen, L., Manion, L., \& Morrison, K. (2013). Research Methods in Education. Londres: Routledge.

Corona-Lisboa J. (2016). Apuntes sobre métodos de investigación. Medisur, 14(1), Recuperado de http://scielo.sld.cu/scielo.php?script=sci_arttext\&pid=S1727-897X20 16000100016\&lng=es\&nrm=iso\&tlng=es 
Corral, M., Almajano, M., \& Domingo, J. (2008). La encuesta SEEQ como instrumento de mejora continuada: aplicaciones en diferentes contextos universitarios. V congrés internacional: el canvi en la cultura docent universitària, guia del congrés», Lleida. Recuperado de https://upcommons.upc.edu/handle/2117/16014?locale-attribute=en

Cortés, E., Campos, M., \& Moreno, M. (2014). Priorización de las Dimensiones de Evaluación al Desempeño Docente por el Estudiante, en tres Áreas del Conocimiento. Formación universitaria, 7(2), 3-10.

Galbraith, C.S., \& Merrill, G.B. (2012). Predicting student achievement in universitylevel business and economics classes: Peer observation of classroom instruction and student ratings of teaching effectiveness. College Teaching, 60(2), 48-55.

García, J., \& Medécigo, A. (2014). Los criterios que emplean los estudiantes universitarios para evaluar la in-eficacia docente de sus profesores. Perfiles educativos, 36(143), Recuperado de http://www.scielo.org.mx/scielo.php?script=sci_arttext\&pi $\mathrm{d}=$ S0185-26982014000100008

García-Señorán, M., González, S., \& Soto, J. (2015). Estudio exploratorio de intereses y motivación para la ejecución de tareas en alumnado de Educación Primaria de la provincia de Pontevedra. Revista de Investigación en Educación, 13(2). Recuperado de http://reined.webs.uvigo.es/ojs/index.php/reined/article/viewFile/1068/361

Glazerman, S., Loeb, S., Goldhaber, D., Steiger, D., Raudenbush, S., \& Whitehurst, G. (2010). Evaluating teachers: the important role of value-added. Recuperado de www. brookings.edu/research/reports/2010/11/17-evaluating-teachers

Instituto de Perfeccionamiento y Estudios Superiores (2009). Investigación Cuantitativa. Recuperado de http://ipes.anep.edu.uy/documentos/investigacion/materiales/inv_ cuanti.pddf

Kane T. J., \& Staiger D. O. (2012). Gathering feedback for teaching: Combining high-quality observations with student surveys and achievement gains. Bill and Melinda Gates Foundation. Recuperado de http://metproject.org/downloads/MET_Gathering_ Feedback_Research_Paper.pdf

Marsh, H. (1982). SEEQ: A reliable, valid, and useful instrument for collecting students' evaluations of university teaching. British Journal of Educational Psychology, 52(1), 77-95.

Marsh, H. (1987). Students' evaluations of university teaching: Research findings, methodological issues, and directions for future research. International journal of educational research, 11(3), 253-388.

Marsh, H., \& Roche, L. (1997). Making students' evaluations of teaching effectiveness effective: The critical issues of validity, bias, and utility. American Psychologist, 52(11), 1187 - 1197.

Martínez, J. (2013). Combinación de mediciones de la práctica y el desempeño docente: consideraciones técnicas y conceptuales para la evaluación docente. Pensamiento Educativo, Revista de Investigación Educacional Latinoamericana, 50(1), 4-20.

Ochoa-Meza, G., Sierra, J., Pérez-Rodrigo, C., \& Aranceta-Bartrina, J. (2014). Validación del cuestionario Pro Children Project para evaluar factores psicosociales del consumo de fruta y verdura en México. Salud Pública de México, 56(2), 165179. Recuperado de http://www.scielo.org.mx/scielo.php?script=sci_arttext\&pi $\mathrm{d}=$ S0036-36342014000200004 
Olavarrieta, J., Gómez, M., \& García N. (2015). Estudio sobre el uso de cuestionarios de opinión de alumnos sobre profesores como método de evaluación docente en una universidad privada de México. Revista de evaluación educativa, 3(2) Recuperado de http://revalue.mx/revista/index.php/revalue/article/view/114/173

Pérez-Poch, A. (2006). Las técnicas de Aprendizaje Cooperativo mejoran y consolidan la calidad docente en la asignatura "Telemática" de EUETIB. Trabajo presentado en XXII Jornadas sobre la Enseñanza Universitaria de la Informática (JENUI 2016). Universidad de Almería. Recuperado de https://www.upc.edu/rima/es/ grupos/grapa-evaluacion-1/recursos-1/aportaciones-del-grupo/antoni-perezevaluacion-con-esat-herramienta-de-soporte-al-aprendizaje-de-telematica/ antoni-perez-poch-las-tecnicas-del-aprenedizaje-cooperativo-mejorando-yconsolidando-la-calidad-docente-en-la-asignatura-telematica-de-euetib

Pintrich, P. (2003). A motivational Science Perspective on the Role of Student Motivation in Learning and Teaching Contexts. Journal of Educational Psychology, 95(4), 667-686. doi: 10.1037/0022-0663.95.4.667

Pintrich, P., \& Schunk, D. (2002). Motivation in education: theory research, and applications. Upper Saddle River: Prentice Hall.

Pounder, J. (2007). Is student evaluation of teaching worthwhile? Quality Assurance in Education, 15(2), 178- 191.

Rama, S. (2011). Professor's performance for effective teaching (Kosovo case). ProcediaSocial and Behavioral Sciences, 12, 117-121. doi: 10.1016/j.sbspro.2011.02.015

Reyero, D. (2014). Teaching excellence at the University. Analysis and proposals for better evaluation of university teacher. Educación XX1, 17(2), 125-143. doi: 10.5944/ educxx1.17.2.11482

Rodríguez, G., \& Ibarra, M. (Eds). (2011). E-Evaluación orientada al e-Aprendizaje estratégico en Educación Superior. Madrid, España: Narcea.

Rueda, M. (2008). La evaluación del desempeño docente en las universidades públicas de México. Revista iberoamericana de evaluación educativa, 1(3). Recuperado de http://www.ses.unam.mx/integrantes/uploadfile/mrueda/Rueda2008_ DesempenoDocenteEnUniversidades.pdf

Rueda, M., \& Luna, E. (2011). La valoración del desempeño docente en las universidades. En M. Rueda (Coord.), ¿Evaluar para controlar o para mejorar? Valoración del desempeño docente en las universidades (pp. 9-30). México: IISUE-UNAM/Bonilla Artigas Editores.

Salazar, J. (2008). Diagnóstico preliminar sobre evaluación de la docencia universitaria: Una aproximación a la realidad en las Universidades públicas y/o estatales de Chile. Revista Iberoamericana de Evaluación de Chile, 1(3). Recuperado de http://www.rinace. net/riee/numeros/vol1-num3_e/art5.pdf

Santigosa, R., Costas, T., Campos, J., \& Jorba, H. (2014). Ser buen docente: ¿qué opinan los estudiantes de la Universidad de Barcelona? Revista iberoamericana de psicología y salud, 5(2), Recuperado de https://dialnet.unirioja.es/servlet/articulo?codigo $=4762654$

Soberón, J., Mirabent, J., Carmona, A., Vidal, O., Carrillo, M., Mercade, L., \& Corral, M. (2015). Las habilidades sociales del docente universitario: Una formación hacia la competencia interpersonal. Revista del Congreso Internacional de Docència Universitària i Innovació, 2, Recuperado de http://www.cidui.org/revistacidui/index.php/cidui/ article/view/816/786 
Solé-Pareta, J., Domingo-Pascual, J., \& Santos-Boada, G. (2014). Actividades para fomentar las competencias transversales relacionadas con el razonamiento científico-técnico. Trabajo presentado a en las XX Jornadas XX Jornadas de Enseñanza Universitaria de la Informática, Oviedo. Recuperado de http://upcommons.upc.edu/bitstream/ handle/2099/15402/P427so_acti.pdf

Tirado, F., Miranda, A., \& Sánchez, A. (2007). La evaluación como proceso de legitimidad: la opinión de los alumnos. Reporte de una experiencia. Perfiles educativos, 29(118). Recuperado de http://www.scielo.org.mx/scielo.php?script=sci_arttextypid=S018526982007000400002 ylng=esynrm=iytlng=es

Verdugo, M., \& Cal, M. (2010). Valoración de la enseñanza: SEEQ. Revista de Formación e Innovación Educativa Universitaria, 3(4). Recuperado de http://refiedu.webs.uvigo. es/Refiedu/Vol3_4/REFIEDU_3_4_2.pdf

Villa, A. (2008). La excelencia docente. Revista de educación, número extraordinario 2008, Recuperado de http://www.revistaeducacion.mec.es/re2008/re2008_08.pdf

Fecha de recepción: 9 de junio de 2016.

Fecha de revisión: 3 de septiembre de 2017.

Fecha de aceptación: 3 de septiembre de 2017. 
